\title{
A Intelligent Campus Based on the Internet of Things------Campus Intelligent Drainage System
}

\author{
Lukai Tong ${ }^{*}$ \\ China Jiliang University, Hangzhou 310018, China. E-mail: 14886906@qq.com
}

\begin{abstract}
Nowadays, the Internet of Things is being used in various fields. Drainage is placed at the top of the priority in campus life. However, the current campus drainage system cannot meet people's yearning for a better life, so the system needs to be optimized. The optimized drainage system is divided into four parts. Sensor part, communication part, cloud control part, water storage/drainage part, identification part. RFID and AI technology are used for identification, temperature, humidity and pressure sensors are used for perception, cloud computing technology is used for cloud control, WIFI technology is used for communication and water storage and drainage control has three modes: remote control, automatic closed-loop control and manual control. Drainage network with circular pipe network drainage system, to achieve intelligent, accurate goals.
\end{abstract}

Keywords: Internet of Things; Intelligence Campus; Drainage System; Supervise

\section{Introduction}

As the development of Internet of Things technology, it has been used in all fields: digital urban management call center, fine agriculture, landscaping, food traceability, smart grid, smart transportation, smart logistics, telemedicine monitoring, smart home. The Internet of Things is all about everything being connected to each other, and its trend is to be applied to every aspect of life, which is relevant to us. And drainage, it's a city life crucial part. With the continuous progress of urbanization, urban agglomerations gradually increase. As urban drainage system planning lags far behind urban development, frequent floods occur ${ }^{[1]}$. For stormwater storage and management, a much faster, more efficient drainage system is needed. In this paper, the existing campus drainage system $^{[2]}$ is optimized by using Internet of Things technology.

\section{Campus drainage control system}

\subsection{A general introduction to the drainage system}

The traditional campus drainage system is mainly drained by two ways, one is the sunken rainwater green space, the other is discharged to the municipal wastewater for centralized treatment through the drainage pipeline. If there is an artificial lake in the campus, part of the drainage will also be accumulated by the artificial lake to relieve the pressure of drainage ${ }^{[3]}$. Based on the traditional drainage method, the new drainage system is improved in terms of speed and intelligence, and the system is divided into five parts: perception part, communication part, cloud control part, water storage/drainage part, and identity identification part. The design drawing is shown in Figure 1. The lake water is

Copyright (C) 2020 Lukai Tong

doi: $10.18686 /$ utc.v6i4.97

This is an open-access article distributed under the terms of the Creative Commons Attribution Non-Commercial License

(http://creativecommons.org/licenses/by-nc/4.0/), which permits unrestricted non-commercial use, distribution, and reproduction in any medium, provided the original work is properly cited. 
mainly used to relieve the pressure of urban campus drainage, and the green space is used as the "sponge" in the concept of "sponge city" to absorb rainwater. Based on a certain control algorithm, the cloud makes an analysis of the lake, green space and weather conditions, and adjust the drainage strategy. The control system is in the full charge of the drainage system team, and others have no right to modify the cloud data, so identity authentication is very critical. The interaction between modules is shown in Figure 2.

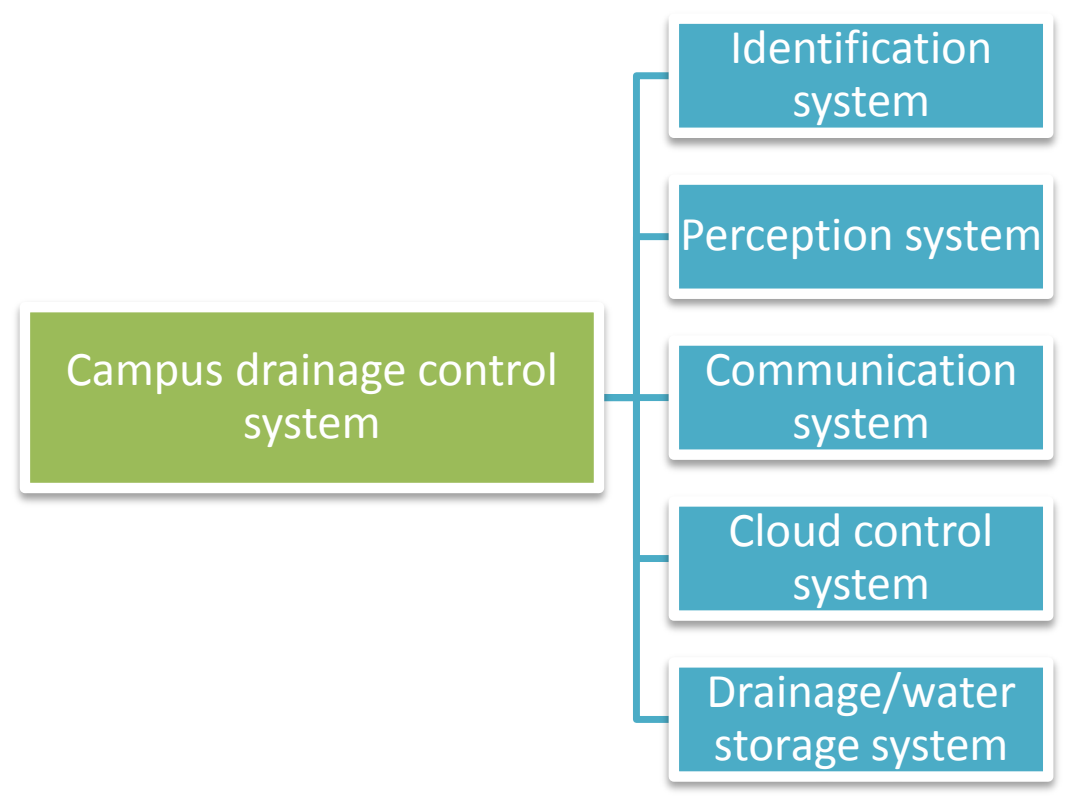

Figure 1. General block diagram of drainage system.

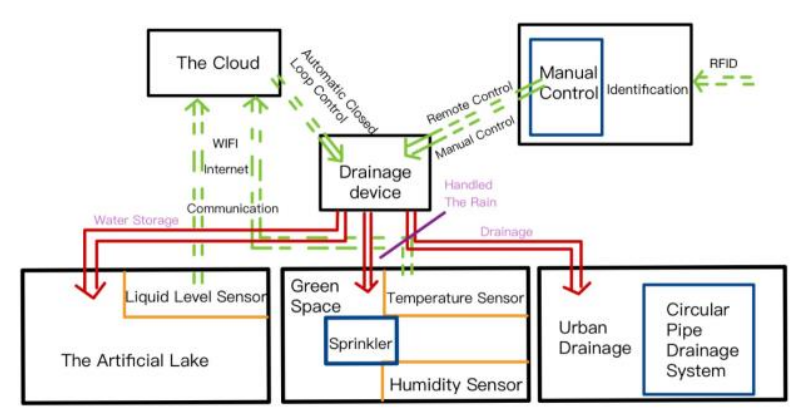

Figure 2. System interaction diagram.

\subsection{Drainage system details}

\subsubsection{Identification system}

(1) RFID technology:

The identity identification system mainly uses RFID technology. It is often embedded in the item or on the item label for its identity. Tags play a vital role in identifying the owner's attributes, such as personal information or related features. Due to the limitation of physical hardware, RFID identification is different from general identification. RFID uses a pseudo-random number generator and a one-way hash function to generate infor- mation. With the popularity of electronic tags, various authentication protocols have been introduced. Lightweight RFID protocol will be adopted in the campus drainage system ${ }^{[4]}$.

(2) The application of RFID:

How to identify the identity information securely and ensure the specification of the authority of the drainage system is the most important. In practice, the identification system is divided into two modules: transmitting module and reading control module. The transmitter module is an instant transmitter, which is embedded in the card. The identity manager can establish the connection with the reading control module through 
his/her card. The reading control module is composed of reader and application system. The reader can send a signal, and once the passive tag receives the signal, it will send a response to respond, so as to realize the data transmission, and then verify the identity.

(3) AI (Artificial Intelligence):

Intelligent control is the highest level control technology of automatic control direction. Artificial Intelligence is an interdisciplinary subject, including machine learning, neural network intelligent control and other fields. It is an open complex intelligence system, whose openness is reflected in the exchange of information between external environment or other systems. It is packaged and integrated by a number of modules, which can have their own functions. Cooperation can become a system to complete a huge project.

(4) AI face recognition technology:

There are two main applications of artificial intelligence face recognition, one is identity verification: prove that you are you; the other is face retrieval: know who I am. And in the identification of identity information, the need to use is the first kind-identity verification. The system administrator input his own face into the system in advance. When the operation permission is needed, face comparison authentication can be carried out. The computer calculates the similarity between the current person and the person in the database through a certain algorithm ${ }^{[5]}$. In order to achieve $100 \%$ identity accuracy, face recognition results are sent to RFID through the interface to prevent misjudgment. After face recognition is passed, it is necessary to enter a password for verification $^{[6]}$. This method enables the administrator to control the system even if the card is lost or not around.

\subsubsection{Perception system}

\section{(1) Sensor technology:}

Pressure sensors ${ }^{[7]}$ are used to measure the liquid level height. As the pressure sensor is a non-contact sensor, it is more convenient than the mechanical and static measurement methods.

Temperature sensors ${ }^{[7]}$ and humidity sensors ${ }^{[7]}$ are used to detect the moisture content index of weather. Temperature sensor changes the resistance value of characteristic resistance caused by the change of temperature. Humidity sensor influences the change of resistance value through the change of ion concentration in the air, which will eventually lead to the change of cir- cuit output voltage or current.

(2) Application of sensor technology:

The capacitive sensor is more suitable for campus drainage system because of its simple structure and good temperature stability. Through the data transmitted from the pressure sensor, the real-time liquid level height of the lake is obtained through simple mathematical calculation, and the data is sent to the cloud through the communication system. The temperature and humidity sensors are thermocouple sensors and capacitive humidity sensors. Through the feedback voltage and current of the sensor in the circuit, the specific real-time temperature and humidity can be obtained through simple mathematical calculation, and the information can be sent to the cloud.

\subsubsection{Cloud control system}

(1) Cloud computing technology:

The main feature of cloud computing technology is the ability to share and access collective resources. Through the cloud, you can have almost unlimited storage space and powerful computing power. With the continuous acquisition of weather data, each region will have their regularity of climate change, according to the temperature and humidity of communication system to collect information, combined with the local weather data, can realize the weather numerical cloud computing services, so that you can avoid costly price to buy a high performance computer, do save resources

(2) Cloud computing applications:

In order to use high-performance computers, various components of numerical weather are encapsulated in the web for ready access, and GWT technology is used to realize the cloud interface of cloud computing web. Through numerical calculation by a large computer, the equations of thermodynamics in the process of weather evolution can be solved, and the corresponding forecast of the weather for a period of time in the future can be made. This method is also known as numerical weather forecast.

\subsubsection{Communication system}

(1) WIFI technology based on STM32:

WIFI communication needs to be based on hardware module. In this paper, STM32 single-chip microcomputer with Cortex-M3 kernel is selected as the core, which belongs to high-performance processor. By configuring certain circuits, hardware modules can be 
formed. In this paper, wM-G-MR-09 single-chip solution $^{[8]}$ is selected, which has high cost performance, high stability and relatively simple hardware design. It is very suitable for application in campus drainage system. When MR-09 works, the transmission rate is independent of the power consumption, so when the transmission rate is increased, the power consumption of the system is constant. In order to save development costs, the SDIO interface can be selected for MR-09 communication in the master control unit. With $88 \mathrm{~W} 8686$ as the core, MR-09 supports dual frequency bands of $2.4 \mathrm{~g}$ and $5 \mathrm{G}$ with high integration.

(2) Application of WIFI technology based on STM32:

In the campus drainage system, the transmitter of the main data to be transmitted is the data detected by the sensor system, as well as the real-time picture and historical data transmitted by a part of the video surveillance system. The receiver of the data is the cloud and the user. Through WIFI communication protocol, the data is sent to the cloud through the Internet for information collection and processing, and the results are sent to the control module for control. The system administrator can also conduct remote control based on the data returned.

\subsubsection{Water storage and drainage control system}

(1) Control mode of campus drainage system:

According to the characteristics of densely populated campus with more green space and man-made lakes, the control is divided into three different modes: manual control, automatic closed-loop control and remote con$\operatorname{trol}^{[9]}$.

When manual control is used for emergency or drainage system maintenance, the system administrator can start and stop the drainage system directly on the control panel after identification.

Automatic closed-loop control is used for general control. Send the data back from the cloud to the programmable controller, and the water pump can adjust the drainage system according to the real-time situation ${ }^{[10]}$. Even through cloud data calculation, the water pump can make judgment on the future rainfall situation and issue instructions to the drainage system in advance.

Remote control is a kind of control method that the administrator can control the drainage system in real time even when he is not on site. Remote intervention of each unit of the drainage system through WIFI technology.

(2) According to the campus drainage system structure:

The purpose of a man-made lake is to store water and relieve the pressure of drainage. Some of the drainage will flow into the man-made lake. The green space is used to absorb rainwater and realize the idea of "sponge campus". The underground drainage adopts the method of distributional drainage. Rainwater flows into the artificial lake and sewage flows into the sewage pipeline. Due to the characteristics of densely populated campus, concentrated residential areas and concentrated water places, the annular pipe network drainage system is adopted $^{[11]}$.

\section{Conclusion}

The intelligent campus drainage system based on the Internet of Things innovatively applies the technology of the Internet of Things into the drainage system. More efficient and accurate control is realized than the previous drainage system. However, this drainage system needs a lot of high and new technology, so it will be more expensive in production cost. At present, its popularization is limited, and it still needs some funds from the state subsidy.

\section{References}

1. Wu D, Zhan S, Li Y, et al. New trends and practical research on the sponge cities with Chinese characteristics. China Soft Science 2016; (1): 79-97.

2. Chen Y. Study on campus rainwater garden design under the concept of sponge city (in Chinese) [Master's thesis]. Nanjing: Nanjing Normal University; 2019.

3. Wang Y. The reasearch on multi-functional stormwater management practices based on campus site characteristics [Master's thesis]. Fuzhou: Fujian Agriculture and Forestry University; 2015.

4. Jiang Z, Huang C, Li L, et al. Application and security challenges of artificial intelligence face recognition technology (in Chinese). Secrecy Science and Technology 2019; (9): 18-22.

5. Qin H, Li T, Guo H, et al. Case study on application of face recognition technology in the library. Journal of Academic Libraries 2018; (6): 49-54.

6. Xie X, Zhu G, Xie W. Fundamentals of Internet of Things technology (in Chinese). Beijing: Tsinghua University Press; 2014.

7. Sun C. The research and implementation of cloud computing service of numerical weather prediction 
system [Master's thesis]. Changsha: National University of Defense Technology; 2009.

8. Chen X, Jiang R, Liu J, et al. The application of cloud computing in numerical weather prediction. The Journal of New Industrialization 2016; (10): 66-69.

9. Yan X. Research and design of WIFI video transmission based on STM32 [Master's thesis]. Taiyuan: North University of China; 2016.
10. Wang Y, Tang H. The analysis and research of "Sponge Campus" drainage system problem- - A case study of the North Campus of Anhui Jianzhu University. Architecture \& Culture 2016; (4): 242243.

11. Wang S. Optimization study of urban drainage system [Master's thesis]. Liaoning: University of Science and Technology Liaoning; 2016. 